\title{
The Palomar Transient Factory Survey Camera: 1st Year Performance and Results
}

\author{
N.M. Law ${ }^{a, b}$, R. G. Dekany ${ }^{b}$, G. Rahmer ${ }^{b}$, D. Hale ${ }^{b}$, R. Smith $^{b}$, R. Quimby ${ }^{c}$, E.O. Ofek ${ }^{c}$, M.

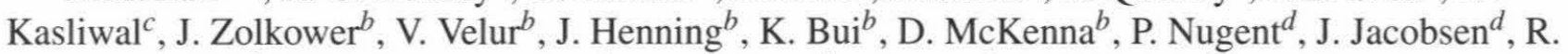 \\ Walters $^{b}$, J. Bloom ${ }^{e}$, J. Surace ${ }^{f}$, C. Grillmair $f$, R. Laher ${ }^{f}$, S. Mattingly $f$, S. Kulkarni ${ }^{c}$ \\ ${ }^{a}$ Dunlap Institute for Astronomy and Astrophysics, University of Toronto, 50 St. George Street, \\ Toronto M5S 3H4, Ontario, Canada; \\ ${ }^{b}$ Caltech Optical Observatories, MS 105-24, California Institute of Technology, Pasadena, CA 91125, \\ USA; \\ ${ }^{c}$ Cahill Center for Astrophysics, California Institute of Technology, Pasadena, CA, 91125, USA \\ ${ }^{d}$ Lawrence Berkeley National Laboratory, Berkeley, CA 94720, USA; \\ ${ }^{e}$ Department of Astronomy, University of California, Berkeley, CA 94720-3411, USA;

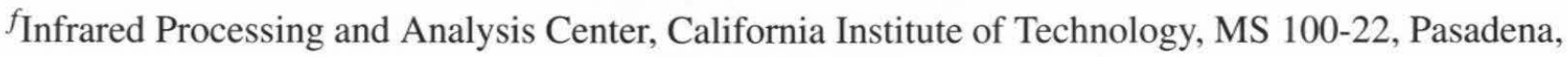 \\ CA 91125, USA;
}

\begin{abstract}
The Palomar Transient Factory (PTF) is a new fully-automated, wide-field survey conducting a systematic exploration of the optical transient sky. The transient survey is performed using a new 8.1 square degree, 101 megapixel camera installed on the 48-inch Samuel Oschin Telescope at Palomar Observatory. The PTF Camera achieved first light at the end of 2008, completed commissioning in July 2009, and is now in routine science operations. The camera is based on the CFH12K camera, and was extensively modified for use on the 48 -inch telescope. A field-flattening curved window was installed, the cooling system was re-engineered and upgraded to closed-cycle, custom shutter and filter exchanger mechanisms were added, new custom control software was written, and many other modifications were made. We here describe the performance of these new systems during the first year of Palomar Transient Factory operations, including a detailed and long term on-sky performance characterization. We also describe lessons learned during the construction and commissioning of the upgraded camera, the photometric and astrometric precision currently achieved with the PTF camera, and briefly summarize the first supernova results from the PTF survey.
\end{abstract}

\section{INTRODUCTION}

The Palomar Transient Factory ${ }^{1,2}$ (PTF) is a fully-automated, wide-field survey aimed at a systematic exploration of the optical transient sky. The transient survey is performed using a new 8.1 square degree camera installed on the 48-inch Samuel Oschin telescope at Palomar Observatory (henceforth P48); colors and light curves for detected transients are obtained with the automated Palomar 60-inch telescope (henceforth P60). PTF uses eighty percent of P48 and fifty percent of P60 telescope time. Four major experiments are being performed during the five-year project: 1) a 5-day cadence supernova search; 2 ) a rapid transient search with cadences between 90 seconds and 1 day; 3 ) a search for eclipsing binaries and transiting planets in high-cadence data; and 4) a $3 \pi$ sr deep $\mathrm{H}$-alpha survey. PTF provides automatic, realtime transient classification and followup, as well as a database including every source detected in each frame. PTF is a collaboration between Caltech, Columbia University, Las Cumbres Observatory, Lawrence Berkeley National Laboratory, Weizmann Institute of Science, and the University of Oxford.

PTF's survey camera ("the PTF Camera") is a $2 \times 6$ CCD array with 101 megapixels, 1-arcsec sampling and an 8.1 square-degree field of view ( 7.26 square-degrees are light sensitive). The camera was extensively re-engineered by Caltech

Further author information: send correspondence to law@di.utoronto.ca 


\begin{tabular}{ll} 
P48 survey camera & CCD array \\
\hline CCD specs & 2K $\times 4 \mathrm{~K}$ MIT/LL 3-edge butted CCDs \\
Component CCDs & 12 CCDs; 1 non-functional \\
Array Levelling & Flat to within 20 microns \\
Pixels & 15 microns/pixel; 100\% filling factor \\
Chip gaps & Median 35 pixels (35 arcsec) \\
Readout noise & $<12 \mathrm{e}^{-}$ \\
Readout speed & 35 seconds, entire 100 MPix array \\
Linearity & $\begin{array}{l}\text { better than 0.5\% up to 60K ADUs } \\
\text { maximum 7 arcsec at array corners } \\
\text { Optical distortion }\end{array}$ \\
& (easily correctable) \\
\hline
\end{tabular}

PTF survey characteristics

\begin{tabular}{ll}
\hline Telescope & Palomar 48-inch $(1.2 \mathrm{~m})$ Samuel Oschin \\
Camera field dimensions & $3.50 \times 2.31$ degrees \\
Camera field of view & 8.07 square degrees \\
Light sensitive area & 7.26 square degrees \\
Plate scale & 1.01 arcsec $/$ pixel \\
Efficiency & $66 \%$ open-shutter (slew during readout) \\
Sensitivity (median) & $\mathrm{m}_{\mathrm{R}} \approx 21$ in $60 \mathrm{~s}, 5 \sigma$ \\
& $\mathrm{m}_{\mathrm{g}^{\prime}} \approx 21.3$ in $60 \mathrm{~s}, 5 \sigma$ \\
Image quality & 2.0 arcsec FWHM in median seeing \\
Filters & $\mathrm{g}^{\prime} \&$ Mould-R; other bands available \\
\hline
\end{tabular}

Table 1. The specifications of the PTF Camera, updated from those previously published ${ }^{1}$ to include recent upgrades to the CCD electronics and camera software.

Optical Observatories for faster readout and robust, low-cost survey operation. The camera was upgraded with a new precision shutter and filter-changer assembly, while to reduce operations cost the camera cooling system was swapped from a $\mathrm{LN}_{2}$ system to a CryoTiger closed-cycle cooler. The CCD readout system was enhanced to double array readout speed (to 30 seconds), and an new software control system was built for the camera. The P48 telescope was refurbished, and a new queue-scheduling automated Observatory Control System (OCS) and Data Quality Monitor (DQM) were implemented for PTF operations.

The full PTF camera system was achieved first light on 13 Dec 2008. A six-month whole-PTF commissioning period followed, during which the camera, telescope and ancillary systems were extensively tested along with the off-mountain data transfer system, data reduction pipelines, and supernova detection and followup strategies. The system was declared fully commissioned on 26 July 2009 , by which time full science operations had started and the PTF collaboration had discovered 108 supernovae in images from the PTF camera.

In section 2 we detail previously-unpublished upgrades applied to the PTF camera and the P48 telescope during the commissioning process. In section 3 we detail the first year on-sky performance of the camera, including the results of long-term image-quality monitoring. Section 4 describes the current photometric and astrometric precisions achievable with the camera, and section 5 summarizes some of the key PTF science results to date.

\section{THE PTF CAMERA ON THE P48 TELESCOPE}

The PTF camera is described in detail in previous ${ }^{1,3}$ papers. Briefly, the system consists of: 1 ) a $12 \mathrm{~K} \times 8 \mathrm{~K}$ mosaic made up of twelve $2 \mathrm{~K} \times 4 \mathrm{~K}$ MIT/LL CCID20 CCDs arranged in a $6 \times 2$ array in a dewar cooled by a CryoTiger closed-cycle cooler; 2) a dual split blade shutter system; and 3) a custom filter changer assembly that allows motorized selection of one of two filters. In typical PTF operations SDSS-g and Mould- $\mathrm{R}^{*}$ filters are mounted. The full current specifications of the PTF camera CCD array and electronics are given in table 2 . Below we briefly describe systems installed and upgraded since the PTF Camera's first light and not described in detail in previous publications.

\subsection{Camera Waveform and Electronics Upgrade}

During the commissioning phase of the camera several artifacts were seen on the various CCDs at high signal levels: reduced full well, image smearing, signal turndown after deep saturation (saturated stars turned to donuts), or simple ADC clipping. The first-light performance was sufficient for initial science operations, but not ideal.

To address these problems an extensive campaign was undertaken to optimize the readout waveforms on a CCD by $\mathrm{CCD}$ basis. The image smearing was solved by adjusting the time delay between the last parallel shift and the beginning of the serial shifting. The signal turndown was found to be due to an overflow of the Summing Well (SW), which has lower capacity than the serial register. At high signal levels the serial shift occurring between CCD reset and sampling of the reset level caused charge to overflow onto the sense node, thus elevating the baseline. To remove this problem the Output Gate and Reset Gate voltages were adjusted to enhance SW capacity while the parallel clocks were tuned to obtain the

\footnotetext{
*approximately equivalent to SDSS-r
} 

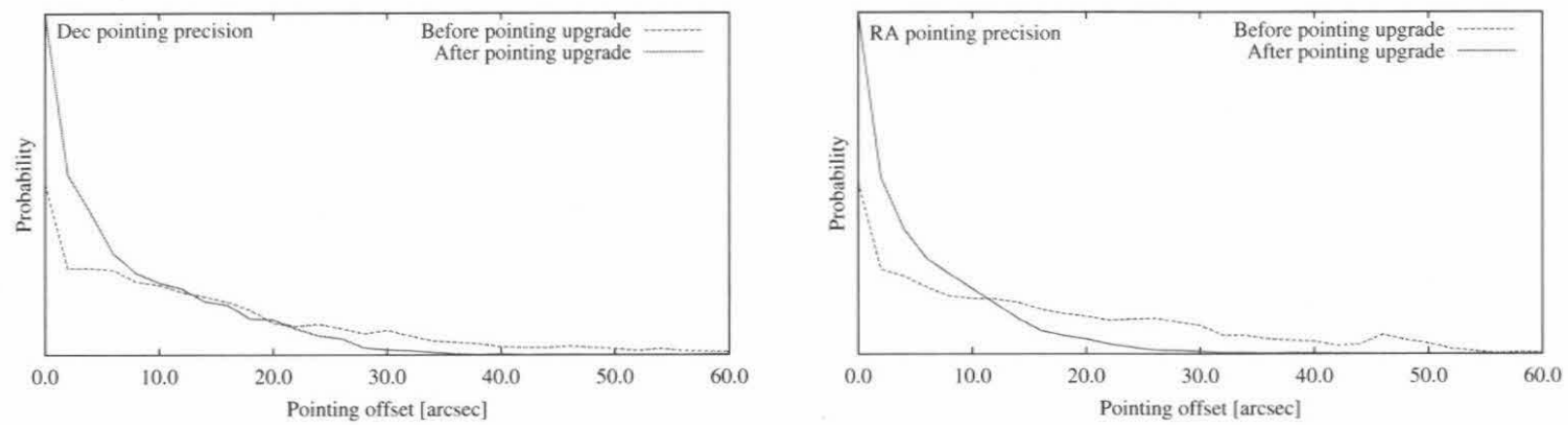

Figure 1. Pointing precision before (red dashed lines) and after (blue solid lines) the P48 pointing upgrade. Calculated from the center displacements from median pointing for 868 fields and 11,676 total measurements before the upgrade, and 692 fields and 14,148 total measurements after the upgrade.

highest well capacity, but not so high as to cause SW overflow or the trapping associated with surface full well. Gain and offset were adjusted to assure that blooming occurred before ADC saturation. All these parameters were adjusted with a goal of preserving charge conservation across saturation, allowing initial low-precision photometric calibration using the brightest stars in the field (i.e. those that have good photometry in all-sky catalogs).

\subsection{Dry Air System}

The PTF camera was designed with a feed to blow dry air across the CCD window, preventing condensation on the cold window. During commissioning an oil film was found to slowly build up on the camera's cold CCD window. The oil was traced to contamination in the legacy air drying equipment. The compressed air treatment system was rebuilt with new filters, new membrane and coalescing dryers, new air lines, and a dewpoint sensors to measure the relative humidity of the dry air. In addition to replacing the treatment system components, a new air purge line was placed between the shutter and filter. The new air line was installed to create a chamber of clean, dry air between the filter and shutter, thus reducing the window's exposure to untreated air when the shutter is open. During this work we found it to be very important to replace as much of the system as possible simultaneously, including the air lines, to avoid cross-contamination of new and old components.

\subsection{P48 Pointing Performance}

An upgraded P48 telescope control system was implemented, including a limited re-write of the TCS code to allow a 13-coefficient pointing model. The upgraded system greatly improved the P48 pointing precision, to 7" and 12" RMS precision in RA and Dec respectively (figure 1), well within PTF specifications.

\section{PERFORMANCE DURING THE FIRST YEAR OF PTF OPERATIONS}

The PTF survey system has been in routine science operation since the Spring of 2009. During that time the PTF camera has taken 48,378 science exposures, for a total of $9.04 \mathrm{~TB}$ of image data. On a typical good-weather night 200-300 images are acquired, depending on the length of the night and the survey in progress. In this section we investigate the on-sky performance of the camera over the first year of PTF operations.

\subsection{Image Quality}

In individual images the camera delivers FWHMs around 2 arcseconds (in median seeing), consistent to $10 \%$ across the entire field of view (figure 2). A histogram of all the image sizes delivered by the PTF camera during the first year of observations is shown in figure 3. The median FWHM delivered meets the system design specification, and during exceptional nights the image quality can be better than 1.5 arcseconds. With 1 arcsecond pixels 1.5 arcsecond PSFs are undersampled and it would be counterproductive to improve the image quality still further. 

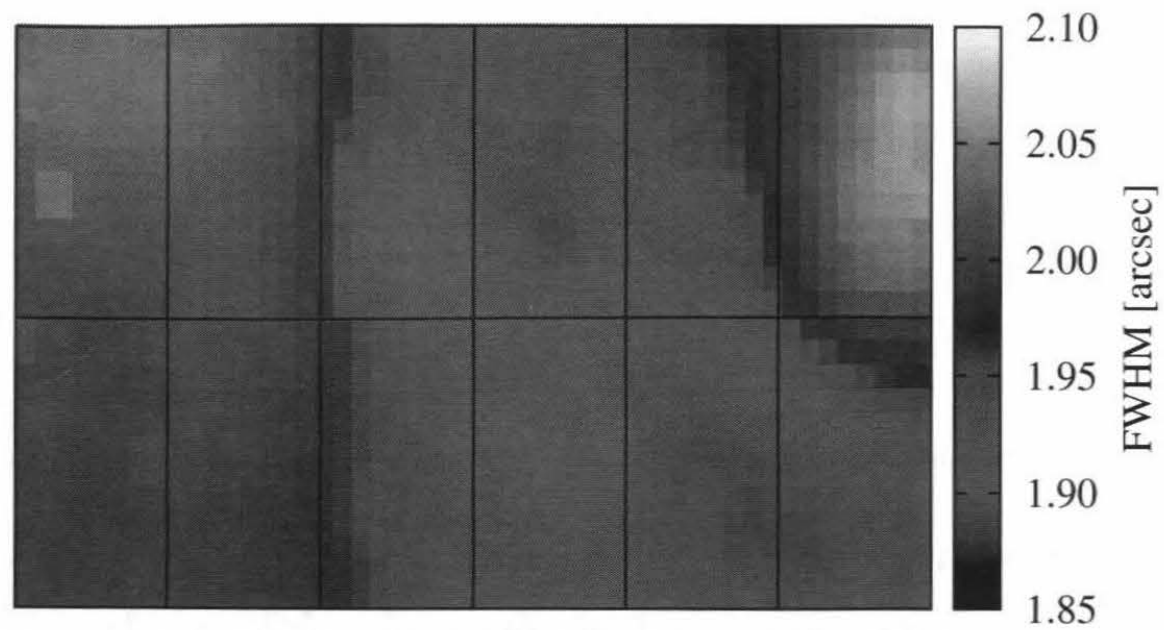

Figure 2. FWHM map for a typical recent R-band PTF camera image, based on SExtractor ${ }^{4}$ FWHM measurements of 10,080 stars across the CCD array. Image FWHM measurements are interpolated over the unresponsive CCD for ease of interpretation. The residual FWHM variations are on the order of 0.2 arcseconds and are due to the $20 \mu \mathrm{m}$ flatness precision of the CCD array, and slightly decreasing optical performance at the edge of the field of view.

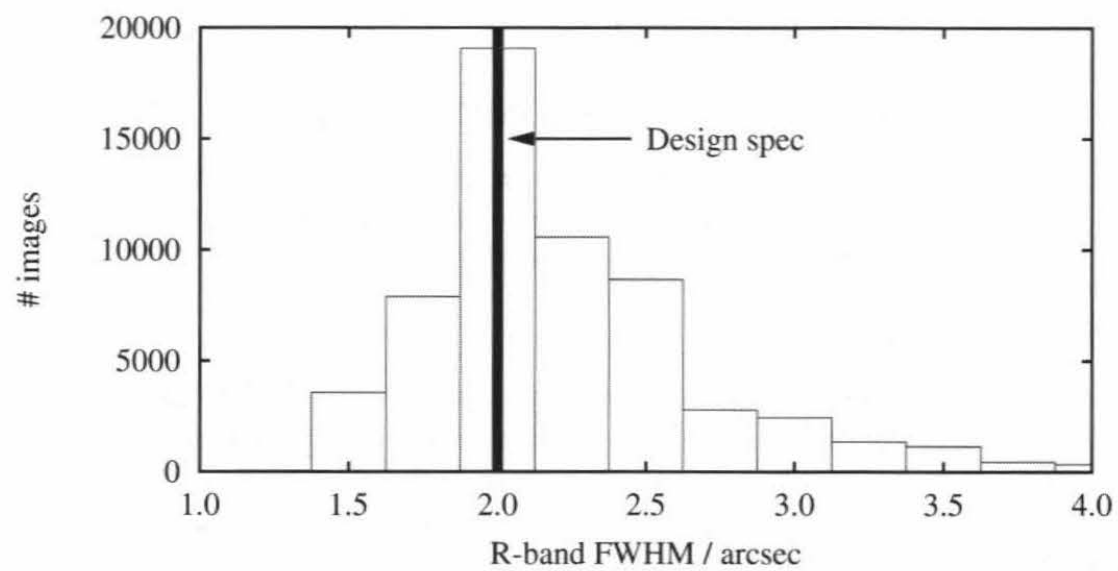

Figure 3. R-band FWHMs delivered by the PTF camera, for all airmass < 1.2 PTF data since June 182009 . The design specification of 2.0 arcsecond FWHMs during median Palomar seeing is also shown. Each chip from the PTF camera is treated as a separate image in this plot, but the variations in FWHM between chips are small (figure 2). Note the tail to larger FWHMs due to nights with poor seeings.

\subsection{Limiting Magnitudes}

The PTF survey's first-year limiting magnitudes are shown in figure 4. The system's design performance is actually improved on during observations near zenith on cloudless, moonless nights (suggesting that the specifications assumed during design were somewhat conservative). For all PTF data, irrespective of moon position or airmass, the limiting magnitudes achieved are also well within the design specifications. Most PTF images in the first year were taken in the R filter; the smaller g-filter sample shows similar performance.

\subsection{Observing efficiency}

The observing efficiency of the P48 camera system is automatically tracked on a nightly basis. During commissioning the survey efficiency was currently approximately $50 \%$ open-shutter time, compared to a goal (and theoretical maximum) of $63 \%$. The reduced efficiency was determined to be the result of multiple small software inefficiencies in the camera and 

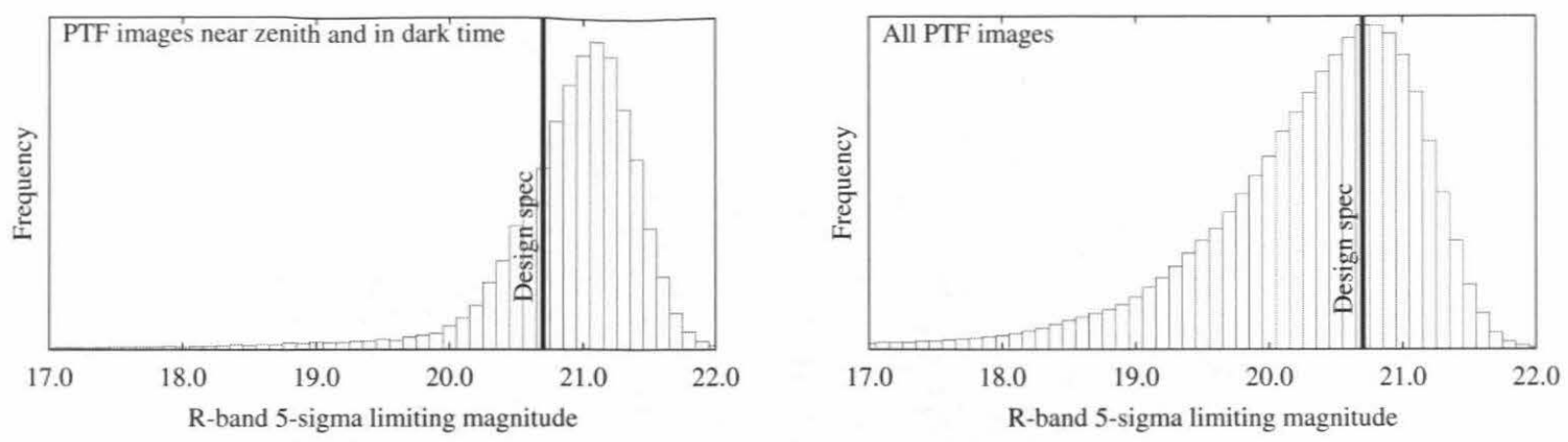

Figure 4. $5 \sigma$ limiting magnitudes for PTF images taken since 18 June 2009, output by the LBNL PTF pipeline reduction system. Top: PTF images taken at airmass $<1.2$ and with the moon below the horizon, for comparison with the PTF camera's design specification for median seeing. We do not attempt to remove images taken during cloudy conditions, hence the long tail towards poorer limiting magnitudes. Bottom: the limiting magnitudes achieved for all PTF data, irrespective of airmass, moon position or weather. This curve most closely matches the performance that can be expected during routine PTF survey observations.

scheduling system. The survey efficiency has been continually improving and in recent months has achieved the theoretical maximum efficiency (one 60-second exposure plus readout time every 96 seconds), although some survey optimization is still required to avoid occasional long slews between targets.

\section{PHOTOMETRY AND ASTROMETRY WITH THE PTF CAMERA}

In this section we characterize the photometric and astrometric precision currently achieved with the PTF camera. Ongoing work is likely to improve the performance further. The photometric and astrometric reduction techniques discussed below are being applied to the output of the final PTF imaging pipeline.

\subsection{Photometric Performance}

Figure 5 shows the R-band photometric precision currently achieved using the PTF camera over long periods. Several photometric reduction procedures are currently under evaluation for the PTF photometric databases. The method shown here optimizes the zeropoints of each image in a group of exposures by minimizing the average RMS photometric variability for all sources in the field, weighted by the average $\mathrm{S} / \mathrm{N}$ of the sources. Other methods under evaluation also achieve similar photometric precisions.

The current-best precision achieved over several month timescales is $\approx 5$ millimags, and stars fainter than $\mathrm{m}_{\mathrm{R}} \sim 16$ are limited by photon statistics. No detrending has been applied to this data, so some of the residual systematic noise is due to inter-night variations. Similar photometric reductions performed on single nights of data reach 2-3 millimag precisions (figure 6).

\subsection{Astrometric Performance}

The current PTF camera astrometric precision for a typical observation is shown in figure 7. The astrometric solutions are generated using SCAMP, ${ }^{5}$ with 5 th-order polynomial distortion correction and using all stars with $\mathrm{S} / \mathrm{N}>10$. The PTF camera internal precision for high $\mathrm{S} / \mathrm{N}$ sources is approximately 70 milliarcseconds, or $\approx 2 \%$ of a pixel. The RMS displacement between PTF and SDSS-DR7 astrometry is $\approx 100$ mas, as expected given the PTF internal precision and SDSS's astrometric precision. 


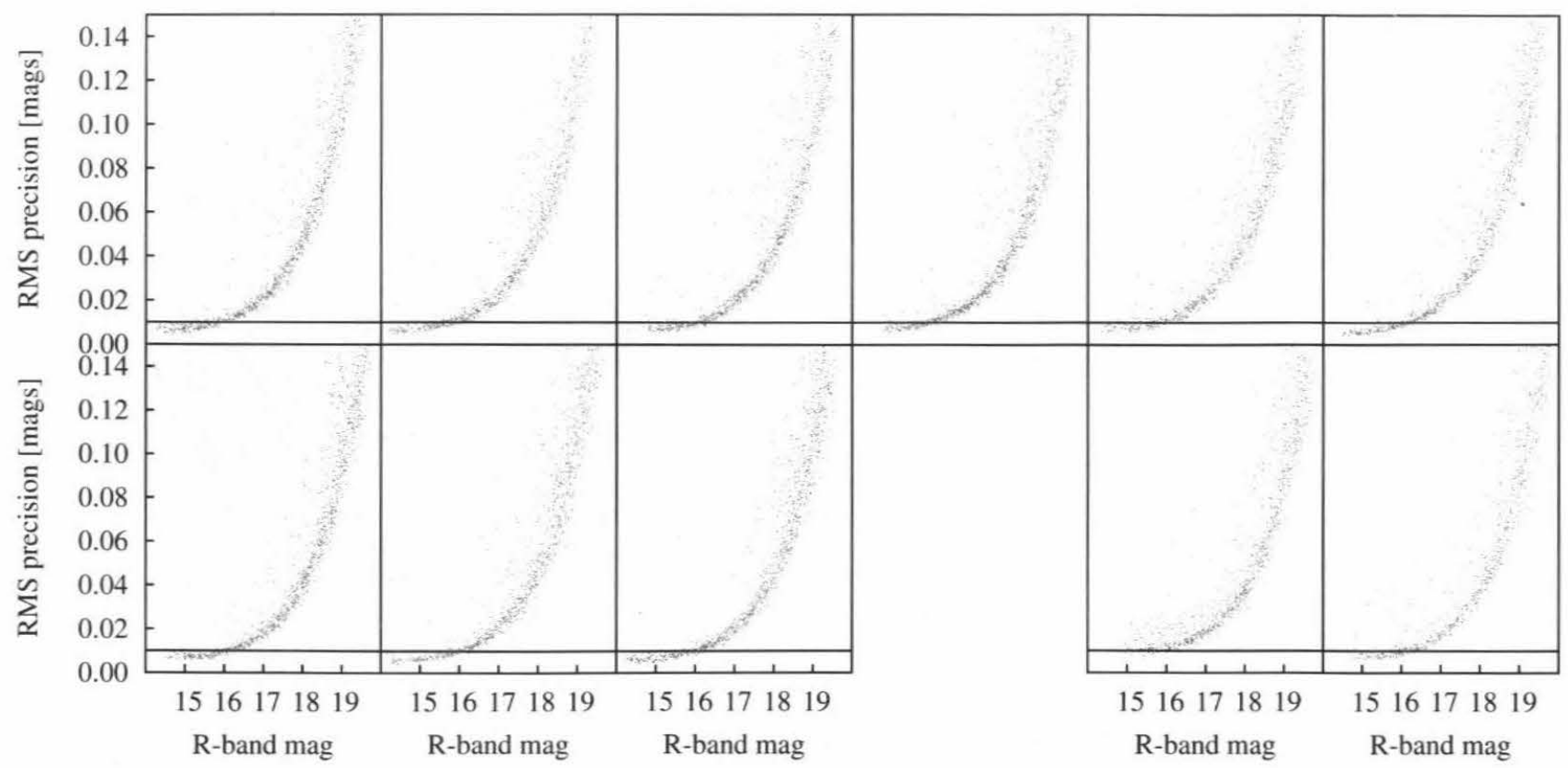

Figure 5. RMS Photometric precision achieved for 194 R-band observations covering 3.5 months targeted at a single PTF field. Individual points correspond to RMS measurements for individual stars. The boxes correspond to different chips in the camera's CCD array (the missing box is due to the inoperative chip). $1 \%$ precision is shown as a line in each box. Note the many variable stars, visible as points with higher-than-expected RMS variability.

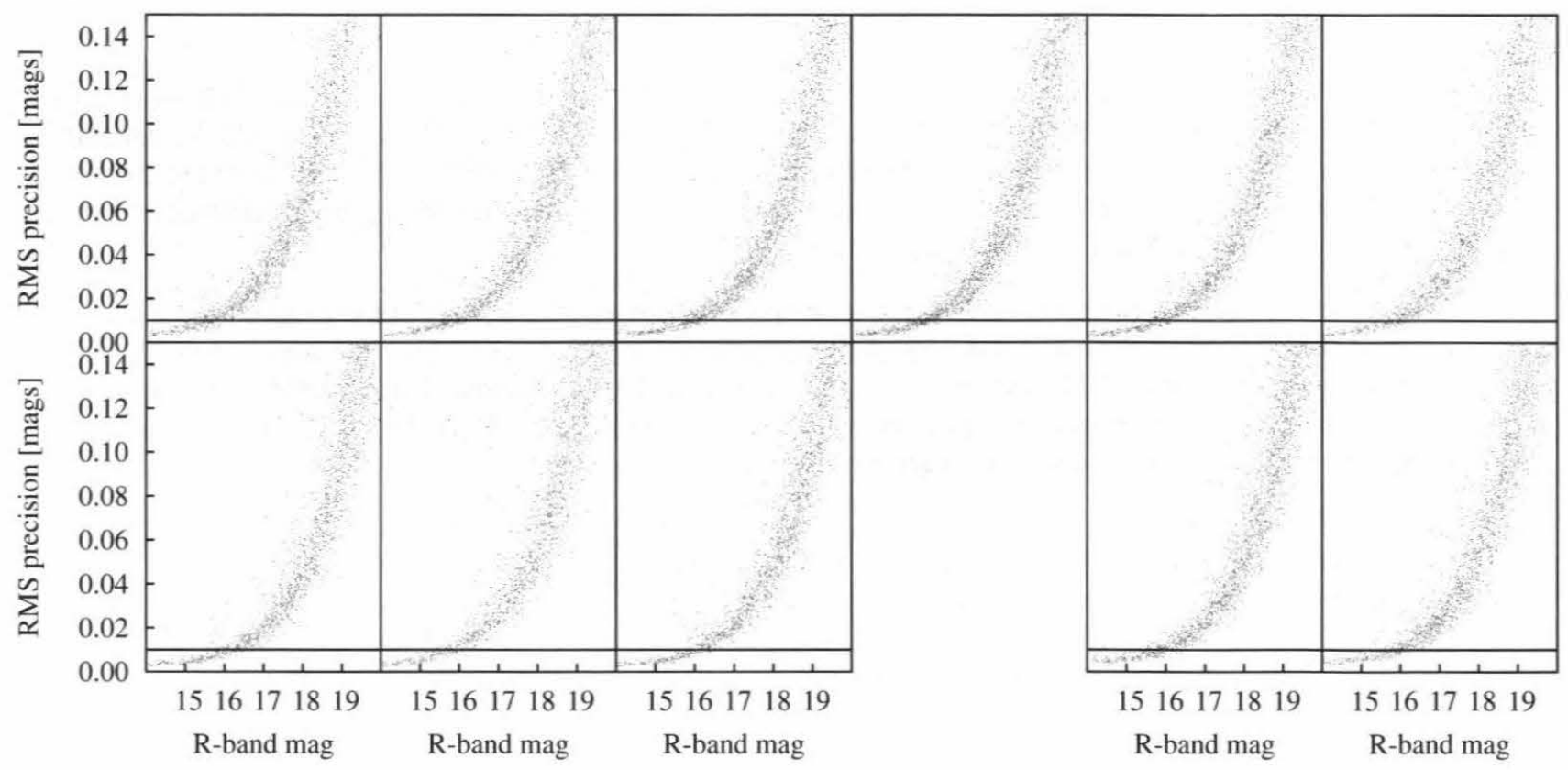

Figure 6. RMS Photometric precision achieved for 24 R-band observations of a typical PTF field taken over 5.3 hours during a single night (airmasses between 1.01 and 2.17 ). The precision for the brightest stars is $\sim 2$ millimags. 

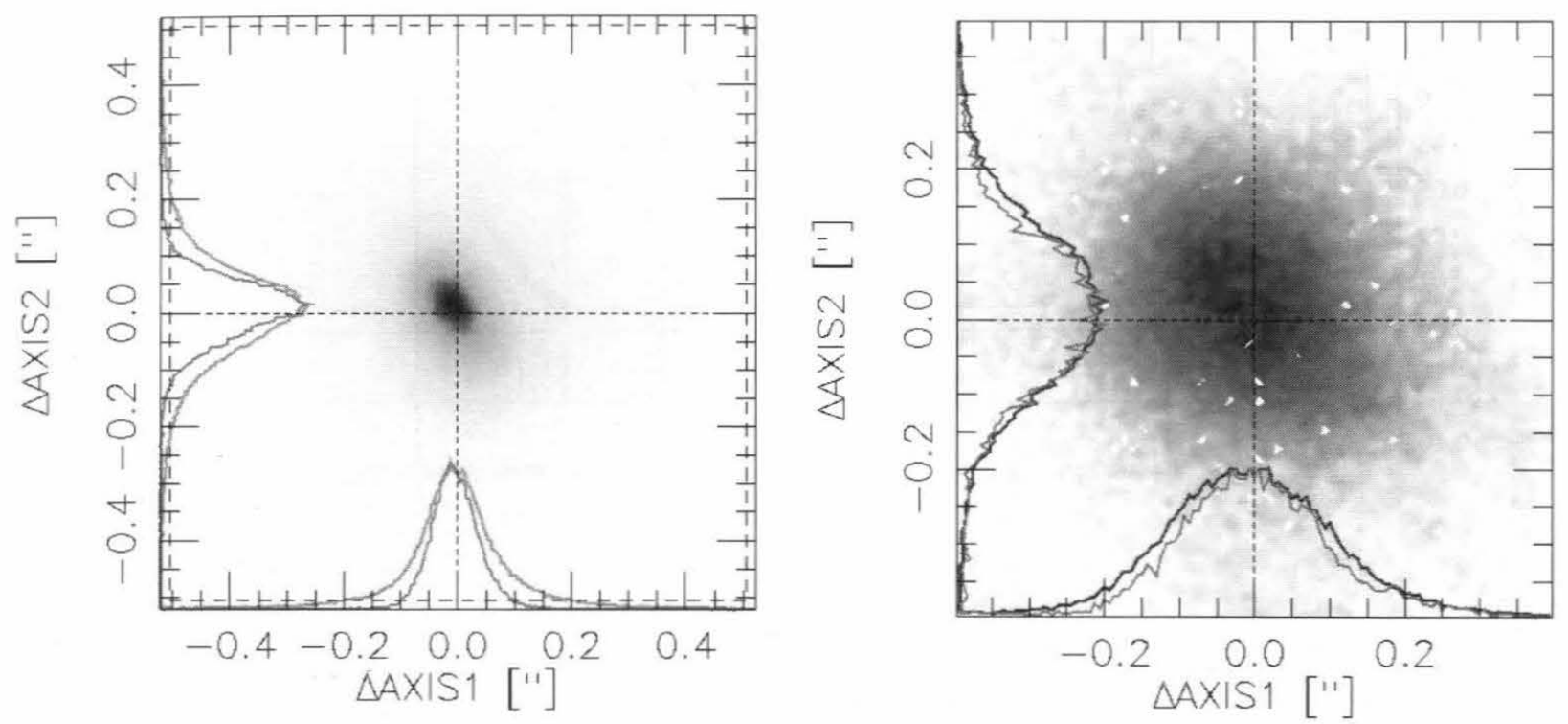

Figure 7. Astrometric precision achieved for 57 exposures of a single PTF field taken over two months (results from a single representative chip are shown). Left: internal precision for all exposures during the run. Right: external precision, comparing to SDSS-DR7.

\section{RECENT RESULTS FROM THE PTF SURVEY}

PTF is already producing a wide variety of science results. The PTF collaboration has (up to 1 June 2010) discovered and spectroscopically confirmed 485 new supernovae ${ }^{\dagger}$, averaging to just over one supernova discovered per night. This discovery rate is increasing with improving survey efficiency and good summer weather; 107 supernovae were discovered in April and May 2010, $\approx 1.8$ supernovae per night. Although it is beyond the scope of this paper to describe the progress of PTF's ongoing Key Projects in detail, we here summarize some recent key results.

- The first compilation of 72 core-collapse supernovae (SNe) from PTF has been used to study their observed subtype distribution in dwarf galaxies compared to giant galaxies. ${ }^{6}$ The nature of the PTF survey provides a minimally biased sample, rich in SNe from dwarf hosts, with spectroscopic classifications. With 15 events detected in dwarf galaxies, the results are still limited by small-number statistics. However, several interesting trends emerge including more core-collapse $\mathrm{SNe}$ in dwarf galaxies than expected.

- A primary science driver of PTF is building a complete inventory of transients in the local Universe (distance less than $200 \mathrm{Mpc}$ ). PTF10fqs ${ }^{7}$ is a transient in the luminosity "gap" between novae and supernovae. Located in the spiral arm of Messier 99, PTF10fqs is red, slowly evolving and has a spectrum dominated by intermediate width $\mathrm{H} \alpha$ and Calcium lines. The explosion signature is similar to M85OT2006-1, SN2008S and NGC300-OT. The origin of these events is shrouded in mystery, controversy (and in some cases, in dust).

- Recent discoveries suggest entirely new pathways for the deaths of massive stars, and the observational case for pair-instability supernovae has now been made. Other, unexplained optical transient phenomena have engendered a wide range of models as typified by the long duration and spectroscopically peculiar event SCP06F6 (theories range from a Texas sized asteroid impacting a white dwarf to the eruption of a carbon rich star). Three discoveries ${ }^{8}$ from PTF resolve the mystery of SCP06F6; it is argued that these four events along with SN2005ap reveal the death throes of the most massive stars - pulsational pair-instability outbursts.

In addition to the supernova results, other PTF Key Projects (including exoplanets and stellar astronomy) have papers in preparation and are actively persuing targets.

†331 SN Ia; 25 SN Ib; 115 SN II; and 14 anomalous SN 


\section{SUMMARY}

The PTF camera has been performing routine science operations since Spring 2009. The system meets or exceeds all the designed image quality, limiting magnitude, and photometric and astrometric specifications. The PTF survey, consisting of the P48 survey telescope and camera, the P60 photometric followup telescope, and numerous spectroscopic followup instruments, is now discovering supernovae at an (increasing) rate of $\approx 2$ supernovae per night.

\section{ACKNOWLEDGMENTS}

We particularly thank the Palomar staff for all their help during the design, setup and operation of the PTF Camera. This paper is based on observations obtained with the Samuel Oschin Telescope as part of the Palomar Transient Factory project, a scientific collaboration between the California Institute of Technology, Columbia University, Las Cumbres Observatory, the Lawrence Berkeley National Laboratory, the National Energy Research Scientific Computing Center, the University of Oxford, and the Weizmann Institute of Science. SRK and his group were partially supported by the NSF grant AST0507734 .

\section{REFERENCES}

[1] Law, N. M., Kulkarni, S. R., Dekany, R. G., Ofek, E. O., Quimby, R. M., Nugent, P. E., Surace, J., Grillmair, C. C., Bloom, J. S., Kasliwal, M. M., Bildsten, L., Brown, T., Cenko, S. B., Ciardi, D., Croner, E., Djorgovski, S. G., van Eyken, J., Filippenko, A. V., Fox, D. B., Gal-Yam, A., Hale, D., Hamam, N., Helou, G., Henning, J., Howell, D. A., Jacobsen, J., Laher, R., Mattingly, S., McKenna, D., Pickles, A., Poznanski, D., Rahmer, G., Rau, A., Rosing, W., Shara, M., Smith, R., Starr, D., Sullivan, M., Velur, V., Walters, R., and Zolkower, J., "The Palomar Transient Factory: System Overview, Performance, and First Results," PASP 121, 1395-1408 (Dec. 2009).

[2] Rau, A., Kulkarni, S. R., Law, N. M., Bloom, J. S., Ciardi, D., Djorgovski, G. S., Fox, D. B., Gal-Yam, A., Grillmair, C. C., Kasliwal, M. M., Nugent, P. E., Ofek, E. O., Quimby, R. M., Reach, W. T., Shara, M., Bildsten, L., Cenko, S. B., Drake, A. J., Filippenko, A. V., Helfand, D. J., Helou, G., Howell, D. A., Poznanski, D., and Sullivan, M., "Exploring the Optical Transient Sky with the Palomar Transient Factory," PASP 121, 1334-1351 (Dec. 2009).

[3] Rahmer, G., Smith, R., Velur, V., Hale, D., Law, N., Bui, K., Petrie, H., and Dekany, R., "The 12Kx8K CCD mosaic camera for the Palomar Transient Factory," in [Society of Photo-Optical Instrumentation Engineers (SPIE) Conference Series ], Presented at the Society of Photo-Optical Instrumentation Engineers (SPIE) Conference 7014 (Aug. 2008).

[4] Bertin, E. and Arnouts, S., "SExtractor: Software for source extraction.," AAPS 117, 393-404 (June 1996).

[5] Bertin, E., "Automatic Astrometric and Photometric Calibration with SCAMP," in [Astronomical Data Analysis Software and Systems XV], C. Gabriel, C. Arviset, D. Ponz, \& S. Enrique, ed., Astronomical Society of the Pacific Conference Series 351, 112-+ (July 2006).

[6] Arcavi, I., Gal-Yam, A., Kasliwal, M. M., Quimby, R. M., Ofek, E. O., Kulkarni, S. R., Nugent, P. E., Cenko, S. B., Bloom, J. S., Sullivan, M., Howell, D. A., Poznanski, D., Filippenko, A. V., Law, N., Hook, I., Jonsson, J., Blake, S., Cooke, J., Dekany, R., Rahmer, G., Hale, D., Smith, R., Zolkower, J., Velur, V., Walters, R., Henning, J., Bui, K., McKenna, D., and Jacobsen, J., "Core-Collapse Supernovae from the Palomar Transient Factory: Indications for a Different Population in Dwarf Galaxies," ArXiv e-prints (Apr. 2010).

[7] Kasliwal, M., Kulkarni, S., Quimby, R., Ofek, E., Nugent, P., Jacobsen, J., Gal-Yam, A., Green, Y., Arcavi, I., Yaron, O., Howell, J., Fox, D., Cenko, S., Kleiser, I., Bloom, J., Miller, A., Poznanski, D., Li, W., Filippenko, A., Starr, D., Law, N., Helou, G., Frail, D., Neill, J., Forster, K., Martin, D., Tendulkar, S., Gehrels, N., Kennea, J., Sullivan, M., Dekany, R., Rahmer, G., Hale, D., Smith, R., Zolkower, J., Velur, V., Walters, R., Henning, J., Bui, K., McKenna, D., and Blake, C., "PTF10fqs: A Luminous Red Nova in the Spiral Galaxy Messier 99," ArXiv e-prints (May 2010).

[8] Quimby, R. M., Kulkarni, S. R., Kasliwal, M. M., Gal-Yam, A., Arcavi, I., Sullivan, M., Nugent, P., Thomas, R., Howell, D. A., Bildsten, L., Bloom, J. S., Theissen, C., Law, N., Dekany, R., Rahmer, G., Hale, D., Smith, R., Ofek, E. O., Zolkower, J., Velur, V., Walters, R., Henning, J., Bui, K., McKenna, D., Poznanski, D., Cenko, S. B., and Levitan, D., "Mysterious transients unmasked as the bright blue death throes of massive stars," ArXiv e-prints (Sept. 2009). 